patients with ongoing alcohol dependence. Extending ACT CNS support across 7 days and deployment of an Assertive Outreach Service should both be considered.

\section{PWE-10 LIVER TRANSPLANT OUTREACH SERVICE: ASSESSMENT OF OUTCOMES SEPT 2019-DEC 2020}

${ }^{1}$ Rebecca Jones*, ${ }^{2} \mathrm{~L}$ Burke, ${ }^{1} \mathrm{M}$ Prince, ${ }^{1} \mathrm{C}$ Lane, ${ }^{1} \mathrm{C}$ Rourke, ${ }^{2} \mathrm{~V}$ Heslop, ${ }^{2} \mathrm{RL}$ Jones ${ }^{1}$ Manchester University NHS Foundation Trust, Manchester, UK; ${ }^{2}$ Leeds Teaching Hospitals NHS Trust, Leeds, UK

\subsection{6/gutjnl-2021-BSG.200}

Introduction Distance from a transplant centre may present a barrier to liver transplant referrals and transplant recipients report disliking travel for long-term follow-up. A liver transplant outreach service based at Manchester Royal Infirmary (MRI) was established collaboratively between the MRI and a transplant centre in Sept 2019. We report the outcomes from this service.

Methods The service runs one day/month and includes posttransplant reviews, transplant assessment triage clinic and a 'working lunch' complex case meeting. Baseline transplant assessment + listing data between April 2017- March 2019 for chronic liver disease indications were collected and compared with those between Sept 2019-Dec 2020, in addition to other service activity data.

Results In the 24 month period in 2017-19 there were 11 chronic liver disease (CLD), excluding HCC, transplant assessments from the MRI. Only 8 were assessed as outpatients. Median referral UKELD was 56. 7/11 were listed with an assessment:listing conversion of $63 \%$.

In the 15 month period Sept 2019-Dec 20 there were 27 pre-transplant reviews with 20 new patients, 4 with HCC and 16 with CLD. One CLD patient had associated HCC. The commonest liver disease indication for review was alcohol related in 8 patients. Nineteen (90\%) patients were reviewed for transplant assessment suitability. 12 (63\%) were referred for assessment with investigations done locally. 9 (75\%) were for chronic liver disease indications and 3 (25\%) were for HCC. Median UKELD was 53.5.

9 assessments have been completed, with 8 wait-listed. None were converted to inpatient assessments. Overall assessment:listing conversion is high: $8 / 9(88.8 \%)$ overall. 3 transplanted to date. 2 HCC patients had bridging therapies locally.

Of 7 not referred for assessment, 1 was suitable for a TIPSS locally, 1 needed pre-habilitation and is under followup, 1 was out-with HCC criteria + is managed locally (still alive). 3 were too advanced/frail + all have since died. 1 was initially thought suitable for assessment but did not progress due to alcohol relapse. During the period April 20-Aug 20 only 1 pre-transplant patient was referred as NHS services were curtailed due to the Covid-19 pandemic.

112 post-transplant reviews have been delivered, in 82 recipients. 98\% gave positive feedback. Post-transplant interventions delivered locally include 2 liver biopsies, 1venogram + the management of 1 patient with acute rejection.

Conclusion Despite the pandemic this service provided improved pre-transplant outcomes in a shorter time frame cf. the preceding 2 years and saved 139 appointments in the transplant centre. The service is efficient, popular with patients, and adds to the case for the widespread adoption of similar partnerships within liver transplant networks.

\section{PWE-11 CAN THE ONLINE SHOPPING MODEL 'LOCK-BOX DELIVERY' IMPROVE HEPATITIS C TREATMENT ACCESS AMONGST VULNERABLE ADULTS?}

${ }^{1}$ Fiona Rees*, ${ }^{1}$ Anja St Clair Jones, 'Margaret O'Sullivan, ${ }^{1,2}$ Sumita Verma. 'Brighton and Sussex University Hospitals Trust, Brighton, UK; ${ }^{2}$ Brighton and Sussex Medical School, Brighton, UK

\subsection{6/gutjnl-2021-BSG.201}

Introduction Hepatitis C virus (HCV) predominantly affects vulnerable and disenfranchised individuals, including people who use drugs (PWUD) and people who are homeless (PWAH); our prior work shows only 5\% attend hospital appointments for HCV management.

Methods The medication homecare delivery model has been used successfully within our Sussex Hepatology Operational Delivery Network (ODN) but relies on clients having an address and/or the ability to sign for deliveries; the latter also proves difficult in hostels where hostel staff are reluctant to be involved with medication deliveries.

On-line shopping companies have adopted 'lock-box' delivery systems for customers who are unable to sign for a delivery, storing the parcel in a secure lock-box thus enabling later convenient collection. We adopted this strategy to ensure safe provision of $\mathrm{HCV}$ medications for difficult-to-engage individuals.

Lock-boxes were installed in two centrally located Brighton hostels that were participating in our on-going communitybased studies; a day hostel (which is accessed by PWUD and PWAH so providing a convenient collection point for these clients) and a residential hostel (which houses PWUD). Lockboxes at the residential hostel enables micro-elimination within the hostel. Access to lock-boxes was via client-specific keycodes with override facilities available for the pilot leads.

Once clients were assessed to be eligible for HCV treatment at the weekly multi-disciplinary ODN meeting, the pharmacy team prescribed and managed delivery to the lock-boxes. Prescribing was enabled via non-medical prescribing pharmacists, cost effective dispensing was guaranteed using the outsourced outpatient pharmacy, medication was dispensed in weekly or monthly blister-packs (depending on client requirements via HCV nurse assessment) and deliveries completed monthly. Usage was monitored on a continuous basis when refilling the lock-boxes and via close relationship with the treating HCV nurse.

Results This pilot ran from January 2020 to April 2020. Seven clients were entered into the pilot. Two PWUD/PWAH and four hostel-resident clients successfully completed HCV treatment. One client decided to disengage from treatment unrelated to the lock-box. The feedback has been overwhelmingly positive from both clients and service providers. The pilot was reliant on one specialised ODN pharmacist. Unfortunately, due to COVID-19 this pilot was put on hold but aims to continue once COVID-19 risks are manageable.

Conclusions Preliminary results from this innovative pilot are favourable as regards improving access to HCV treatment amongst a difficult-to-engage cohort and merits further assessment. 